\title{
Technology of Sound Velocity Correction for Multi- beam Bathymetry Sounding
}

\author{
Weiming $\mathrm{Xu}^{*}$ and Kai Ma \\ Department of Hydrography and Cartography, Dalian Naval Academy 116018, Dalian, Liaoning, P. R. China \\ ${ }^{*}$ Corresponding author
}

\begin{abstract}
The multi-beam echo sounding system was the most effective instrument to detect the seabed topography. In order to reduce the seafloor terrain distortion caused by the representative error of sound velocity profile (SVP), the indirection adjustment inversion method was introduced after analyzing the impact of the sound profile measuring error on the seabed terrain. To testify the proposed indirection adjustment technology, two group experiments were designed, in which Group 1 used the adjustment inversion method to correct the SVP data with the small error while Group 2 utilized the same method to correct the SVP data with the large error. The results of field experimental data are shown that: the standard deviation (STD) of the inversing SVP is reduced by $64.2 \%$ and that of the single Ping seabed topographic reduce $80.7 \%$. The computational efficiency of adjustment inversion method is better than that of the substitute SVP method. Additionally, the presented approach, which can overcome many disadvantages of the manual approach to SVP correction, is superior to that of the substitution SVP and optimizing the structure of SVP.
\end{abstract}

Keywords-multi-beam bathymetry souding; sound correction; seabed topography; indrection adjustment

\section{INTRODUCTION}

Acoustic is the best and often the solo mean to detect the water column and sea bottom efficiently and accurately [1]. A large variety of instruments now available, in these sonar mapping systems which can be divided into three categories, that is, single-beam echo-sounders, multi-beam echo-sounders, and side scan system. Nowadays, the multi-beam sounding system, which integrates the technologies of satellite navigation and positioning, computer science, and the digital sensor etc., is widely used in the domains such as the marine engineering, the underwater archaeology, and the marine national defense construction [2-3]. The velocity at which the acoustics waves travel is also varied with the temperature of the water, its salinity, and whether it contains heterogeneities (e.g., bubbles or suspended sediments). Because it is generally difficult to measure local sound velocity, it is most often derived from proxy measurements of temperature, salinity, and depth, using tools such as XBT (expendable - bath thermograph) or CTD (conductivity - temperature - depth). When even these measurements are not available, approximate values can be derived from compilations such as the Levitus database [1]. Accordingly, the accuracy of multi-beam seabed topography measurement significantly depends on the measuring accuracy of the sound velocity profile (SVP) [3]. Due to the sound velocity would change in space and time as the sea flow, different sound velocity profile structure could also lead to the changes of the acoustic wave propagation, which brings about the distortion terrain of the seabed. Three technologies of sound velocity correction are used to deal with the above-mention distortion [2-5]. The first is based on the substitution of sound velocity profile, namely, the principle of minimal distance or minimal time. However, the correction effect of this method is limited and hard to meet the actual requirement. The second is the artificial modification of some parameters during the data processing system. With the continually increasing volume of data from advanced multibeam sonar systems, the manual editing process has become very time consuming, especially in shallow water surveys. The third is to optimize the structure of simulating a velocity profile based on the sound velocity formula, however, which requires more samples and parameters, and more complicated calculation. Therefore, based on the indirection adjustment theory, the inversion SVP is contrastive analyzed with the equivalent SVP in this paper.

\section{THE IMPACT OF THE SOUND PROFILE ERROR ON THE SEABED TOPGRAPHY}

Using the multi-beam system (MBS) to detect the flat area shallow water would be affected by the complicated marine environment. Consequently, there would be certain errors between the measured sound profile data and the actual sound profile data. The measuring accuracy of sound velocity profile restricted the multi-beam measuring precision of the seafloor topography, which was affected by temperature, salinity, and pressure, etc [6-7].

As shown in FIGURE I, according to Snell's law [1], the entire water could be regarded as a composition of multiple uniform layers of water. Assuming that with $1,2, \ldots, n$ to index each water layer, in the surface of each layer, according to the law of refraction, which is given by 


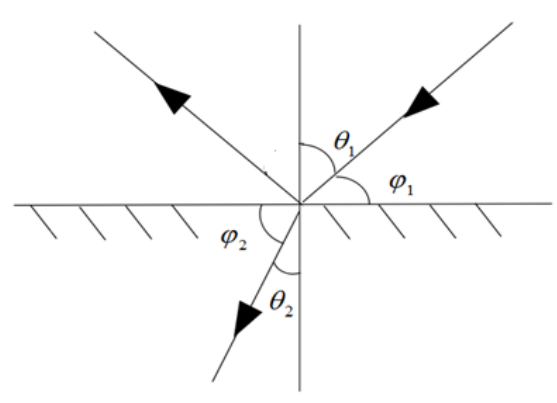

FIGURE I. THE GEOMETRY OF ACOUSTIC REFRACTION

$$
\frac{\sin q_{1 j}}{C_{1}}=\frac{\sin q_{2 j}}{C_{2}}=\ldots=\frac{\sin q_{n j}}{C_{n}}=p_{j}
$$

where $\theta_{i j}$ is the grazing angle after beam pass through each water layer. $C_{i j}$ is referred to as the velocity in each uniform water layer, $\mathrm{i}=1,2, \ldots, n$. $p$ is the Snell constant. According to the Snell's law, after continuously propagating through each multiple layer, it appears that the sound ray turn to be bent. It's obviously that the sound velocity error may interfere with the multi-beam measuring precision due to the sound ray bending. On the edge of the beam, this interference phenomenon is particularly serious [8-10]. The seafloor topography appears as a curving shape on the transverse section, which is referred to as the "frown face", or its symmetric graph, namely, the "smiling face".

\section{INDIRECTION ADJUSTMENT INVERSION METHOD}

The actual seafloor terrain also be regarded as flat, and the aim of the adjustment inversion method is to seek for a set of model correction coefficients to ensure its correctional and actual displacement match optimally. In another words, the terrain distortions can be transform into the actual topography which be supposed to flat by nonlinear weighted least squares (NWLS) principle, and then the inversion SVP that close to the actual SVP is obtained [11-13].

Consider the acquired beam signal travel time $T$ of unidirectional transmission beam in the process of the entire sounding without error. Using adjustment inversion method to correct the sound velocity error and the seabed distortion, assume the vertical displacement of a certain beam is $Z_{j}$, and the horizontal displacement is $X_{\mathrm{j}}$. According to the stratification, we know the heights of layers $n$-1, the sound velocity of each layer, the Snell ray parameters, and the true value of the one-way spreading time $T$ of sound wave. Without considering the errors of surface velocity, we obtain the observation equations by indirect adjustment as follows

$$
X_{j}=F_{j}\left(C_{1}, C_{2} \ldots C_{n}\right)=\sum_{i=1}^{n-1} \Delta_{i j} \cdot C_{i}^{2} p_{j}+\left(T_{j}-\sum_{i=1}^{n-1} \Delta_{i j}\right) C_{n}^{2} p_{j}
$$

$$
Z_{j}=H_{j}\left(C_{1}, C_{2} \ldots C_{n}\right)=\sum_{i=1}^{n-1} \Delta Z_{i j}+\left(T_{j}-\sum_{i=1}^{n-1} \Delta t_{i j}\right) \cdot C_{n} \cos \theta_{n j}(3)
$$

where $i$ is the number of layers, $i=1,2, \ldots, n . j$ is the number of sound beams, $j=1,2, \ldots, m . \Delta t_{\mathrm{ij}}$ is beam travel time in the layer.

The number of observed beam $m$ is often greater than the number of sound velocity parameters, so the equation system has a unique solution. But the observation equations is nonlinear, it needs to be linearized by Taylor series expansion method. Taking $X_{\mathrm{ij}}{ }^{\mathrm{o}}$ and $Z_{\mathrm{ij}}{ }^{\mathrm{o}}$ as the approximation of the vertical displacement true value and the horizontal displacement true value while the sound beam $j$ passing through the layer $i, \mathrm{~d} X_{\mathrm{i}}$ and $\mathrm{d} Z_{\mathrm{i}}$ as the correction of the approximation and the true value, therefore, the linear model of the indirect adjustment function is [14-15]

$$
\begin{gathered}
\hat{X}_{j}=X_{j}^{o}+d X_{j}=F_{j}\left(C_{1}^{o}, C_{2}^{o}, \cdots, C_{i}^{o}\right)+\sum_{i=1}^{n} A_{i j} \hat{c}_{i} \\
\hat{Z}_{j}=Z_{j}^{o}+d Z_{j}=H_{j}\left(C_{1}^{o}, C_{2}^{o}, \cdots, C_{i}^{o}\right)+\sum_{i=L}^{n} B_{i j} \hat{c}
\end{gathered}
$$

where $\hat{C}$ is the true value of sound velocity. $C_{\mathrm{o}}$ is the observed value of sound velocity. $A_{\mathrm{ij}}$ and $B_{\mathrm{ij}}$ are the coefficients of sound velocity correction for the adjustment inversion model, namely, the derivative of the vertical displacement and horizontal displacement on the sound velocity.

Solving simultaneous equations (4) and (5) would get a $m \times n$ Jacobi matrix of $\boldsymbol{A}$ and $\boldsymbol{B}$. The sound velocity correction of partial derivative matrix $\boldsymbol{A}$ and $\boldsymbol{B}$ could get the total matrix $\boldsymbol{D}=\left[\begin{array}{ll}\boldsymbol{A} & \boldsymbol{B}\end{array}\right]^{\mathrm{T}}$. The actual research indicates that some characteristic values of the coefficient of square $D^{T} \boldsymbol{P D}$ is small, and the condition number of equations is large, the inverse square matrix is singular, therefore, the solution tends to diverge. To solve this problem, the damping least-square method [13-15], which adds a damping term after the phalanx $\alpha \mathbf{I}$, is introduced. It is found that the co-factor matrix tends to singularity, and the reasonable damping coefficient value should be established. The method of design selection damping coefficient, currently, the damping coefficient of adaptive selection is still a subject of academic discussion, and the most widely used is ridge-tracing method. Apply the ridgetracing method to determine a set of $\alpha$ value at the starting point 0.5 , the greater the divergence of the co-factor array, the smaller the value. And the corresponding parameter values are listed, and $\alpha$ is the damping coefficient which makes the most stable parameter values.

Thus, the equations to get the sound velocity correction is attained as follows

$$
\widehat{\boldsymbol{c}}=\left(\mathbf{D}^{\mathrm{T}} \mathbf{P D}+a \mathbf{I}\right)^{-1} \mathbf{D}^{\mathrm{T}} \mathbf{P L}
$$


where $\boldsymbol{P}$ is the weight matrix of the observed value. The setting of weight should be considered that the reliability of the edge beam signal data in the multi-beam system is lower than the central beam. According to the precision of the actual measurement for the various sound beams, the lower the accuracy is, the smaller the weight of the beam is. Where $\mathbf{I}$ is the unit matrix, $\alpha$ is the damping factor, the size of which depends on the actual situation, that is, if the coefficient of square $\boldsymbol{D}^{\mathrm{T}} \boldsymbol{P D}$ has a relatively large singularity, then a larger damping coefficient is required. Otherwise, choosing a smaller coefficient, the matrix $\boldsymbol{L}$ is written by [13]

$$
\boldsymbol{L}=\left[\begin{array}{c}
X^{0}-F\left(C^{0}\right) \\
Z^{0}-H\left(C^{0}\right)
\end{array}\right]
$$

And the correction value of the underwater observation value matrix $v$ is [16]

$$
v=[x z]=D \hat{c}-L
$$

Add the distortion correction value to the seabed topography data, based on the central beam measurements to assess its correction accuracy, and assume the iteration threshold is $0.25 \%$ of the depth and central beam depth is actual depth. If the relative error of depth is greater than the iteration threshold, add the sound velocity correction to the original sound profile data again as the initial parameter to calculate, and iterative calculation will be constantly conducting to meet the requirements of International Hydrographic Organization (IHO) and National Oceanic and Atmospheric Administration (NOAA) [6-7].

\section{EXPERIMENTAL DATA AND ANALYSIS}

In order to testify the effectiveness of the proposed technology, the multi-beam sounding data of China East Sea is chosen as the experimental model. In the experimental data the seabed topography appears the distortion caused by the sound velocity error.

The calculation example selects 6 main test lines and 1 inspection line in the sea area, and the depth variation is 49.2 $\sim 63.1 \mathrm{~m}$. The inspection line is mainly used to verify the effect of correction and does not participate in correction. Remove abnormal data and add correction (attitude, tide and time delay, etc.) as experimental preparation.

In this section, two groups of experiment are designed. Group 1 uses the adjustment inversion method to correct the SVP data with the small error and Group 2 uses the same method to correct the SVP data with the large error.

\section{A. The Experimental Group 1}

The number of sampling points for the actual seabed topography is 101 . The obtained parameter $C_{0}$ is $1502 \mathrm{~m} / \mathrm{s}$ and the $g$ is -0.315 . After 35.6 seconds equivalent SVP is calculated. Set the depth of the inspection line to the actual depth.

As shown in FIGURE II, the inversing SVP is svp6 curve, the measuring SVP is svp1 curve, and the replaced SVP is svp2 curve. The single Ping depth correction is shown in FIGURE III, in which data1 is the actual depth, data2 is the correction depth, and data 3 is error depth, respectively. The statistics of terrain correction is listed in TABLE II.

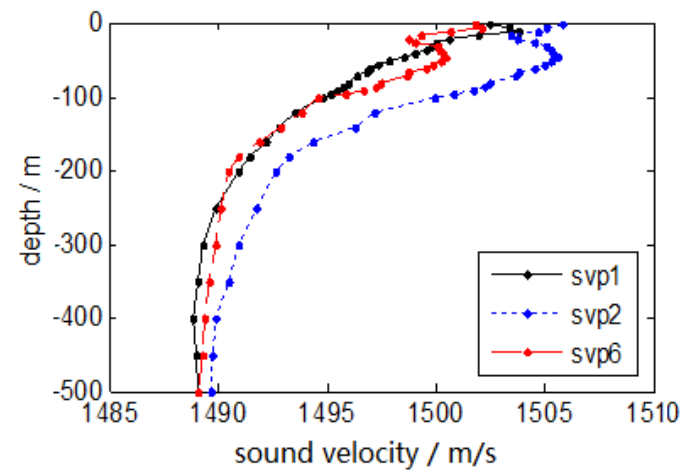

FIGURE II. THE SOUND VELOCITY PROFILE OF GROUP 1

TABLE I. THE ERROR STATISTICS OF GROUP $1 /(\mathrm{m} / \mathrm{s})$

\begin{tabular}{cccc}
\hline Error value & Minimum & Maximum & STD \\
\hline Error SVP & 0.11 & 8.12 & 1.48 \\
Inversion SVP & 0.01 & 3.11 & 0.53 \\
\hline
\end{tabular}

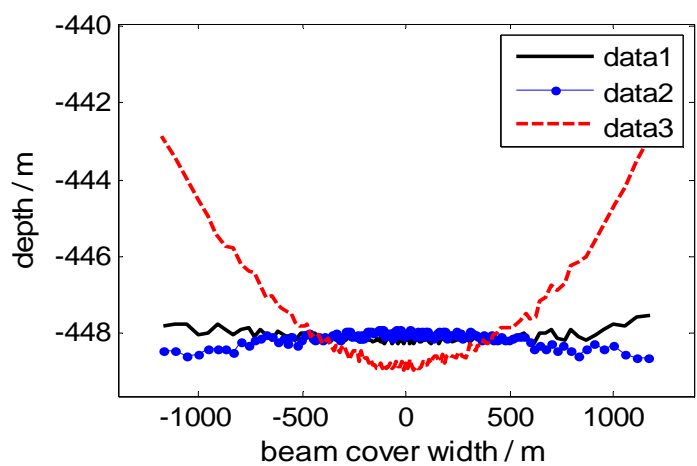

FIGURE III. SINGLE PING CORRECTION OF GROUP 1

TABLE II. TERRAIN CORRECTION STATISTICS OF GROUP 1/ (m)

\begin{tabular}{crrr}
\hline Error value & Minimum & Maximum & \multicolumn{1}{l}{ STD } \\
\hline Error depth & 0.01 & 3.41 & 0.90 \\
Correction depth & 0.01 & 0.46 & 0.37 \\
\hline
\end{tabular}

As shown in TABLE I and TABLE II, The field data results show that the STD error of the inversing SVP is reduced by $64.2 \%$ and that of the single Ping seabed topographic reduce $66.7 \%$. Obviously, the seabed terrain is significantly improved as shown in FIGURE III. 


\section{B. The Experimental Group 2}

The water volume is designed by step, by which, $0 \mathrm{~m}-100 \mathrm{~m}$ each layer $5 \mathrm{~m}, 100 \mathrm{~m}-300 \mathrm{~m}$ each layer $10 \mathrm{~m}$, and $300 \mathrm{~m}-500 \mathrm{~m}$ each layer $20 \mathrm{~m}$. When solving the normal equation, the cofactor matrix tends to singularity, and the singularity is larger. The parameter value diverges to $10^{-3}$, therefore, to search for the damping coefficient in the range of $a=1 \times 10^{-2}$ to $\mathrm{a}=1 \times 10^{-4}$, and get the optimal damping coefficient $a=2 \times 10^{-3}$.

After iteration calculating, the inversion SVP is obtained, then, setting the depth of the inspection line to the actual depth.

As shown in FIGURE III, the inversing SVP is svp7 curve, the measuring SVP is svp3 curve, and the replaced SVP is svp1 curve. The single Ping depth correction is shown in FIGURE V, in which line 1 is the actual depth, line 2 is the correction depth, and line 3 is error depth, respectively. The statistics of terrain correction is listed in TABLE IV.

The field data results show that the STD error of the inversing SVP is reduced by $64.2 \%$ and that of the single Ping seabed topographic reduce $80.7 \%$.

TABLE III. THE ERROR STATISTICS OF GROUP 2 / (m/s)

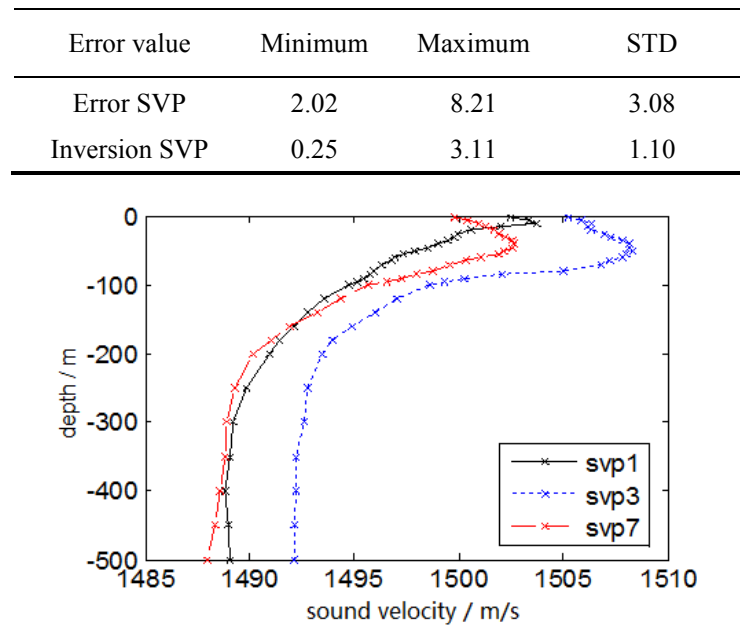

FIGURE IV. THE SOUND VELOCITY PROFILE OF GROUP 2

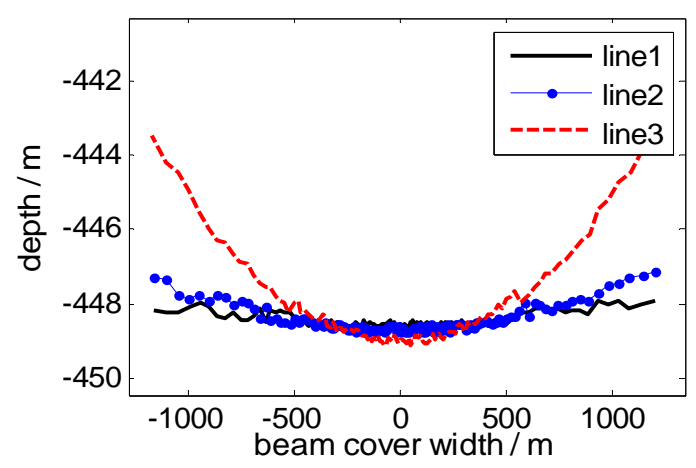

FIGURE V. SINGLE PING CORRECTION OF GROUP 2
TABLE IV. TERRAIN CORRECTION STATISTICS OF GROUP 2/ (m)

\begin{tabular}{cccc}
\hline Error value & Minimum & Maximum & STD \\
\hline Error depth & 0.02 & 4.18 & 2.34 \\
Correction depth & 0.01 & 0.94 & 0.45 \\
\hline
\end{tabular}

\section{CONCLUSION}

This paper first analyzed the main reason of the underwater terrain distortion due to the velocity error in the MBS for the flat area measurement. And then a sound velocity profile and the distortion correction technology of seabed topography are proposed based on indirection adjustment. The field experimental data show that: in the shallow sea water, the inversion sound velocity profile calculated by adjustment method is closer to the real profile compared to the error sound profile. Furthermore, the technology could effectively correct the distortion of the seafloor topography, reduce the depth error, which is satisfied the demand of high precision in the terrain measurement of the shallow sea. Additionally, the presented approach that can overcome many disadvantages of the manual approach to SVP correction, such as: time requirement, repeatability, and human error.

\section{ACKNOWLEDGEMENT}

This work was supported by the National Science Foundation under Grant 61071006, by the National Science Foundation for Post-doctoral Scientists of China (Grant No.2012T5087), by Open Fund of State Key Laboratory of Information Engineering in Surveying, Mapping and Remote Sensing under Grant 10P03.

\section{REFERENCES}

[1] B. Philoppe, The Handbook of Sidescan Sonar. Chichester: Praxis Publishing Ltd, 2009.

[2] R. Dashen, S. M. Flatte, W. H. Munk, et al, Sound Transmission Tthrough A Fluctuating Ocean. UK: Cambridge University Press, 2010.

[3] G. J. Zhan,Y. Z. Ouyang, X. P. Lu, et al, "Comments on the revision of the specifications for hydrographic survey". Hydrographic Surveying and Charting, 2014, 34(1): pp.76-79.

[4] X. P. Lu, S. F. Bian, M. T. Huang, et al, "An improved method for calculating average sound speed in constant gradient sound ray tracing technology". Geomatics and Information Science of Wuhan University, 2012, 37(5) : pp. 590-593.

[5] B.Calder and L. Mayer, Robust Automatic Multi-beam Bathymetric Processing. U.S. Hydrographic Conference. Norfolk, VA, USA, 2001.

[6] NOAA, Hydrogrographic Surveys Specification and Deliverables. 2003

[7] IHO, IHO Standards for Hydrographic Surveys, Special Publication No.44(4 $\left.{ }^{\text {th }}\right)$. International Hydrographic Bureau, 1996.

[8] X. Geng and A. Zielinski "Precise muiti-beam acoustic bathymetry". Marine Geodesy, 1999, 22(3) : pp. 157-167.

[9] R. Hare, Error Budget Analysis for US Naval Oceanographic Office Hydrographic Survey Systems. Final Report for Task 2, FY 01, September 2001.

[10] S. Dan, "On the least-squares adjustment of a straight line". Bulletin Géodésique (1946-1975), 1972, 103(1) : pp.25-30.

[11] J. J. Zhu, T. Y. Zuo, and Y. C. Song, Error Theory and Foundation of Surveying Adjustment. Beijing: Surveying and mapping press, 2013.

[12] B. Schaffrin and Y. A. Felus, On Total Least-Squares Adjustment with Constraints. Berlin: Springer Heidelberg, 2005. 
[13] J. J. Moré, "The Levenberg-Marquardt algorithm: implementation and theory". Lecture Notes in Mathematics, 1978, 630: pp.05-116.

[14] C. L. Lawson and R. J. Hanson, "Solving least squares problems". Siam Review, 2006(3) : pp.518-520.

[15] G. I. Allen, L. Grosenick L, and J. Taylor, "A generalized least-square matrix decomposition". Journal of the American Statistical Association, 2014, 109(505) : pp.145-159.

[16] X. D. Yin, Y. Chen, J. Tang Jun, et al, "A comparison of international standards for hydrographic survey". Hydrographic Surveying and Charting, 2011, 32(4) : pp. 35-38 